\title{
Preliminary Evaluation of Probiotic Potential of Yeasts Isolated from Bovine Milk and Curd of Sri Lanka
}

\author{
D.U. Rajawardana*, I.G.N. Hewajulige ${ }^{1}$, C.M. Nanayakkara ${ }^{2}$, S.K.M.R.A. Athurupana ${ }^{3}$ and \\ W.M.T. Madhujith ${ }^{3}$
}

\author{
Department of Plant Sciences, Faculty of Science \\ University of Colombo \\ Sri Lanka
}

\begin{abstract}
There has been mounting interest in the health benefits associated with live microorganisms commonly known as probiotics. Many probiotic bacterial species have been identified. However, the potential of yeasts as a source of probiotics has not been well explored. The present study was carried out to screen and identify potential probiotic yeasts from selected dairy sources available in Sri Lanka. Yeasts from raw bovine milk and curd were isolated, purified, selected and phenotypically characterized by performing morphological, physiological and biochemical tests. Isolates were assessed for their ability to survive under simulated gastro-intestinal conditions to explore their probiotic potential. Approximately, 190 colonies similar to yeast were isolated and 45 isolates of the division Ascomycota were selected and coded for convenience (SLDY_001-SLDY_045). Most promising probiotic isolates (20) were genotypically identified to be species of Pichia (55\%), Candida (30\%), and Kluyveromyces (15\%) of the family: Saccharomycetaceae. Considering a threshold of $>95 \%$ similarity to the type strain, eight different yeast species were identified. Isolates (SLDY_005, SLDY_006 and SLDY_039) of Kluyveromyces marxianus species showed the highest probiotic potential from the pool. The strain confirmation and in-vitro/invivo safety assessment of these isolates will further verify their suitability as probiotic starter cultures to be used in local food and pharmaceutical industries.
\end{abstract}

Keywords: Curd, gastro-intestinal conditions, Kluyveromyces, probiotic yeast

\section{INTRODUCTION}

Yeasts constitute a large and heterogeneous group of microorganisms included in the kingdom of Fungi. In addition to their role in the food processing industry, yeasts play various roles in livestock feeding, veterinary practices, medical, biomedical and pharmaceutical industries (Jakobsena and Narvhu, 1996). In recent years, yeasts are gaining increased attention from the food industry as probiotics. FAO/WHO (2002) defines probiotics as living microorganisms, which upon ingestion in adequate amounts confer health benefits to the host. Accordingly, any nonpathogenic microorganism capable of surviving in the gastro-intestinal tract (GIT) of the host and provide additional health benefits could be considered as a candidate for probiotic use. While bacterial probiotics are common and

\footnotetext{
1 Food Technology Section, Industrial Technology Institute, Colombo, Sri Lanka

2 Department of Plant Sciences, Faculty of Science, University of Colombo, Sri Lanka

3 Department of Food Science and Technology, Faculty of Agriculture, University of Peradeniya, Sri Lanka

* Corresponding author: deepaniur@gmail.com
} 
mostly studied, yeast probiotics are yet to be explored. When compared with well-known bacterial probiotics, yeasts offer different advantages and are genetically more stable than bacteria. They have more diverse enzymatic profiles and more versatile effects on the immune system, natural resistance against antibiotics and can use for patients undergoing prolonged antibiotic treatments. They also appear to be better suited for nutritional enrichment and delivery of bioactive molecules (Nayak, 2011). Yeasts also have a long history of safe human consumption in traditional fermented foods (Jakobsena and Narvhu, 1996). This includes buffalo curd fermented with indigenous starter cultures. Curd contains numerous undefined species of lactic acid bacteria and yeasts, which act synergistically to develop desirable taste, texture, flavor, aroma and extending the shelf life. As reported by previous researches (Maccaferri et al., 2012), Saccharomyces cerevisiae var.boulardii and kluyveromyces marxianus $\mathrm{B} 0399^{\circledR}$ are live yeasts used extensively as probiotics and often marketed as dietary supplements. Therefore, yeasts are promising candidates for the development of novel probiotics and probiotic products.

Common yeast genera with probiotic properties include Saccharomyces, Pichia, Metschnikowia, Yarrowia, Candida, Debaryomyces, Isaatchenkia and Kluyveromyces (Nayak, 2011). Predominant genera of yeasts found in bovine milk and fermented dairy products also include Saccharomyces, Pichia, Candida, Isaatchenkia, Debaryomyces, Kluyveromyces and Rhodotorula. Therefore, dairy sources could be considered as a unique environment for the selection of novel yeast strains. Despite the occurrence of yeasts in raw bovine milk and many dairy related products and also in human gastrointestinal tract, studies, which examine their probiotic features, are limited. In this backdrop, the present study was carried out with the objective of isolation, subsequent characterization and exploration of probiotic diversity of yeasts present in raw bovine milk and curd manufactured by home based producers using indigenous starters.

\section{METHODOLOGY}

\section{Sample collection, media and chemicals}

Samples (cattle milk and curd, 30 samples each) were collected during October to December 2015 representing three different climatic zones of Sri Lanka as per the statistical methods and procedures in Sri Lanka standard for milk and milk product sampling (SLSI: 1404:2010). Samples collected to sterilized disposable polypropylene tubes $(50 \mathrm{ml})$ were cooled immediately and transported on ice to the laboratory to be stored at $-20{ }^{\circ} \mathrm{C}$ for analysis, for no longer than 3-4 hours. All microbiological media were obtained from Oxoid, UK, chemicals from Sigma, St. Louis, USA and genomic DNA purification Kit (Wizard ${ }^{\circledR}$ ), Promega, USA.

\section{Isolation and morphological characterization of yeast}

Samples were serially diluted using $0.85 \% \mathrm{NaCl}$ and microbial counts were taken by pour and spread plate techniques. Yeast peptone dextrose agar (YPDA), Malt extract-yeast extract-peptone-glucose agar (MYPG) and Potato dextrose agar (PDA) were used for the enumeration of yeasts with $0.1 \mathrm{~g} / \mathrm{L}$ chloramphenicol and incubated aerobically for 5 days at $25{ }^{\circ} \mathrm{C}$. Colonies with distinct morphological differences were selected and purified by repeated streaking on PDA. Isolates were preserved in YPDA slants at $4{ }^{\circ} \mathrm{C}$ and $40 \%$ glycerol stocks at $-20{ }^{\circ} \mathrm{C}$.Colony morphologies (form, size, elevation, margin, texture and 
colony color) were visually examined and then cells were microscopically observed after wet mounting and Methylene blue staining (Barnett et al., 2000).

\section{Biochemical characterization of isolates}

Catalase and Urease test: Catalase producing yeasts were identified by slide method.Commercially available Christensen's urea agar base (Merck) was used to identify urease producing yeasts (Christensen, 1946).

Sugar fermentation test: The fermentation basal medium was prepared using $0.45 \%(\mathrm{w} / \mathrm{v})$ yeast extract; $0.75 \%(\mathrm{w} / \mathrm{v})$ peptone and $2 \%(\mathrm{w} / \mathrm{v})$ of sugars (glucose, sucrose and lactose independently) in distilled water. Fermentation test was carried out as described by Nahvi and Moeini (2004). Conversion of the medium from green to yellow was taken as the positive reaction.

Growth on 50\% glucose: The growth ability of yeasts at high concentration of sugars was tested on media having $50 \%(\mathrm{w} / \mathrm{v})$ glucose, $1 \%(\mathrm{w} / \mathrm{v})$ yeast extract, 3\% agar and chlorampenicol $0.01 \%(\mathrm{w} / \mathrm{v})$.

Liquid assimilation of carbon and nitrogen compounds: Assimilation of carbon compounds was determined using bacto yeast nitrogen base medium without amino acids (Sigma, Y0626), for sucrose, D-maltose, raffinose, L-rhamnose, glycerol and D-mannitol. Nitrogen assimilation was checked using yeast carbon base medium (Sigma, Y3627) for potassium nitrate, L-cysteine and L-lysine (Gadaga et al., 2000).

\section{Survival of yeasts in physiological and simulated chemical conditions existing in the GIT}

Probiotic potential of the isolates was determined by investigating their tolerance to temperature, $\mathrm{pH}$, bile and simulated gastric enzymes following the methods explained by Walker and Gilliland, 1993 and Aswathy et al. (2008). Fresh yeast (18 hours old) cultures were prepared and adjusted to $0.6 \mathrm{OD}$ at $600 \mathrm{~nm}$ (UV-Visible spectrophotometer) to determine the temperature tolerance. Cultures were transferred to 96-well microtiter microplate and incubated aerobically at $10{ }^{\circ} \mathrm{C}, 37{ }^{\circ} \mathrm{C}$ and $45{ }^{\circ} \mathrm{C}$ over 5 hours. After every hour of incubation, samples were periodically drawn out to determine the cell concentration by measuring OD at $600 \mathrm{~nm}$. Tolerance to different $\mathrm{pH}$ levels was studied by incubating the isolates in YPD broth medium adjusted to $\mathrm{pH}$ 1.5, 3.0 and 9 following the method described above. To test the bile tolerance YPD broth medium was prepared by adding $0.3,0.5$ and $3 \%$ of bile (oxgall, Sigma-Aldrich, B-8631). The best bile tolerant isolates were further studied for their tolerance to simulated gastric enzymes by preparing YPD broth medium containing pepsin (3 g/L, Sigma P-7000) and pancreatin (1 g/L, Sigma P-1750) separately, each with 2 different $\mathrm{pH}$ levels (pH 2 and 8).

\section{Genotypic identification of the selected isolates}

Isolates grown in YPD broth medium at $25{ }^{\circ} \mathrm{C}$ for 12 hours were centrifuged, and pallet was washed twice in phosphate-buffered saline (PBS) pH (7.2). Genomic DNA was extracted and purified using the Wizard ${ }^{\circledR}$ Genomic DAN Purification Kit, following the manufacturer's instructions. Selected regions of $18 \mathrm{~S}$ rRNA gene were PCR amplified with universal primers (ITS1 - TCCGTAGGTGAACCTGCGG, ITS4 - TCCTCCGCTTATTGATATGC) and amplified products were subjected to DNA sequencing at Macrogen-South Korea. Resulted 
sequences were analysed using online Basic Local Alignment Search Tool (BLAST) and similarity to the type strain was conformed comparing with the National Center for Biotechnology Information (NCBI) data base.

\section{Statistical Analysis}

Enumerations were done in triplicate, experiments were performed in duplicate and experimental data were expressed as mean \pm standard deviation (SD).

\section{RESULTS AND DISCUSSION}

\section{Enumeration and preliminary selection of Yeasts}

\section{Differentiation of yeasts and molds}

From the three different yeast isolation medias used maximum number of yeasts were enumerated on YPDA. Hence, it was selected as the most suitable growth medium for the isolation of yeast throughout the study. Previous researchers also have reported YPDA as a better enumeration media for the isolation of yeast (Oda and Ouchi, 2000). From the 60 samples analyzed, 190 isolates that resembled yeasts were selected while removing the molds based on their colony morphologies. Subsequently, eighty isolates which were closely resembling yeast and having distinct morphological differences were selected and purified by repeated streaking.

\section{Catalase and Urease tests}

The selected isolates were catalase positive. Yeasts are either aerobes or facultative aerobes; hence production of catalase enzyme is an important criterion for preliminary selection. Predominant genera of yeasts with probiotic affinities found in dairy sources belonging to the division Ascomycota and the production of extracellular urease has been generally considered as a universal character of Basidiomycetous yeasts shared by very few Ascomycetous yeasts (Rij,1984). Rutherford (2014) suggests that urease positive microorganisms have preserved roles in promoting bacterial and fungal infections. Furthermore, enzymatic hydrolysis of urea (milk contains approximately $0.2-0.4 \mathrm{~g} / \mathrm{L}$ ) leads to a slower reduction of $\mathrm{pH}$ during fermentation process (Spinnler and Corrieu, 1989) thus could consider as undesirable for industrial fermentations. Considering above factors 45 distinct urease negative isolates were selected and coded for convenience (SLDY_001SLDY_045) for further characterization.

\section{Colony and cell morphology}

The yeast colonies generally looked similar with fewer variations. Most of the cells appeared oval to elongate in shape, arranged singly, in pairs or in chains/ bunches. Reproduction was by budding or by psedohypha. Isolates with closely similar morphologies were grouped into five categories (Table 1). When compared with the colony and cell morphologies and mode of asexual reproduction (Li et al., 2015), isolates in Group 1 resembles either Saccharomyces sp. or Pichia sp., Group 2, Kluyveromycessp. Group 3 and 4 Candida sp. and Group 5 to Rhodotorulasp. Our categorizations are also similar with the previously reported data of Gadaga et al. (2000) and Kurtzman et al. (2011). 
Table 1. Macroscopic and microscopic features of yeasts isolated from dairy sources

\begin{tabular}{|c|c|c|}
\hline Group & $\begin{array}{l}\text { Macroscopic and microscopic } \\
\text { features }\end{array}$ & Isolate number \\
\hline 1 & $\begin{array}{l}\text { Circular or slightly undulate shape } \\
\text { colonies of large/medium size, } \\
\text { butyrous texture, milky white color, } \\
\text { rough surface, flat with entire } \\
\text { margins.Ovoid to elongate cells } \\
\text { arranged singly or in pairs reproduction } \\
\text { by budding }\end{array}$ & $\begin{array}{l}\text { SLDY_001, SLDY_002, SLDY_004, } \\
\text { SLDY_007, SLDY_008, SLDY_009, } \\
\text { SLDY_010, SLDY_011, SLDY_015, } \\
\text { SLDY_017, SLDY_018, SLDY_021, } \\
\text { SLDY_022, SLDY_025, SLDY_027, } \\
\text { SLDY_028, SLDY_029, SLDY_030, } \\
\text { SLDY_035, SLDY_036 }\end{array}$ \\
\hline 2 & $\begin{array}{l}\text { Circular shape colonies, medium size, } \\
\text { puffy, moist-dull surface, smooth } \\
\text { butyrous texture, grayish-white, raised, } \\
\text { spreading, with } \\
\text { margins.Ovoid/ellipsoidal entire } \\
\text { arranged singly, in pairs or small } \\
\text { clusters or chains. Reproduction by } \\
\text { budding }\end{array}$ & $\begin{array}{l}\text { SLDY_005, SLDY_006, SLDY_019, } \\
\text { SLDY_039 }\end{array}$ \\
\hline 3 & $\begin{array}{l}\text { Circular shape large colonies, soft, } \\
\text { smooth and spongy, butyrous texture, } \\
\text { off-white/white color, raised with } \\
\text { entire margins. Spherical/ } \\
\text { ovoid/cylindrical cells arranged singly } \\
\text { or in pairs. Reproduction by } \\
\text { psedohypha }\end{array}$ & $\begin{array}{l}\text { SLDY_003, SLDY_014, SLDY_023, } \\
\text { SLDY_024, SLDY_026, SLDY_032, } \\
\text { SLDY_033, SLDY_034, SLDY_040, } \\
\text { SLDY_041, SLDY_042, SLDY_043, } \\
\text { SLDY_044, SLDY_045 }\end{array}$ \\
\hline 4 & $\begin{array}{l}\text { Circular shape small/medium colonies, } \\
\text { cream colored, glistening surface, } \\
\text { smooth and spongy, raised colonies } \\
\text { with entire margins.Globose to oval } \\
\text { cells arranged singly or in chains. } \\
\text { Reproduction by budding }\end{array}$ & SLDY_012, SLDY_013, SLDY_016 \\
\hline 5 & $\begin{array}{l}\text { Circular shape small/medium colonies, } \\
\text { pigmented (pink color), glistening } \\
\text { surface, smooth, raised colonies with } \\
\text { entire margins. Globose to ovoid cells } \\
\text { arranged singly or in chains. } \\
\text { Reproduction by budding }\end{array}$ & SLDY_031, SLDY_037, SLDY_038 \\
\hline
\end{tabular}




\section{Biochemical characterization of isolates}

Isolates, namely SLDY_009, SLDY_010, SLDY_024, SLDY_031, SLDY_033, SLDY_034, SLDY_037, SLDY_038 and SLDY_041 either lost their viability or got contaminated while sub-culturing. These isolates were discontinued from further characterizations (Table 2).

\section{Sugar fermentation}

Yeasts gain carbon typically from hexose sugars, such as glucose and fructose or disaccharides such as sucrose and maltose while some species can also metabolize pentose sugars such as xylose, alcohols and organic acids (Ebabhi et al., 2013). However, as mentioned by Gana et al. (2014) and Li et al. (2015), all strains of Pseudozyma and some strains of Debaryomyces sp. are unable to ferment D-glucose whereas Saccharomyces, Pichia, Kluyveromyces, Candida as well as Rhodotorula species easily ferment D-glucose. All tested isolates in the pool were able to ferment D-glucose and their morphologies also tally with the above mentioned D-glucose fermentative types indicating their suitability to consider as isolates belonging to those species. Nearly two third (70\%) of the isolates were able to ferment lactose whereas $30 \%$ could not. Literature reveals that $S$. cerevisiae and some Debaryomyces species are unable to grow and ferment lactose when it is provided as the sole carbon source as it doesn't possess lactose metabolizing system. Lactase or $\beta$-Dgalactosidase enzyme is present in lactose fermenting yeasts such as Kluyveromyces lactis, Kluyveromyces fragilis, and Candida pseudotropicalis (Nahvi and Moeini, 2004). Hence, lactose fermentative yeasts in the pool should also contain above mentioned species which has a potential to grow in milk and whey, therefore could be promising candidates for bio processing industries.

\section{Growth on $50 \%$ glucose}

Except, SLDY_026, SLDY_027 and SLDY_029 other isolates grown well on media supplemented with $50 \%$ glucose thus, majority of the isolates could be considered as suitable candidates for the production of highly concentrated food products. Gana et al. (2014), reported that the Pseudozyma and Cryptococcus species are unable to grow under high osmotic pressure conditions. Based on the colony characteristics and biochemical test results, the pool of yeast isolates belong to Kluyveromyces, Pseudozyma, Cryptococcus, Candida, Rhodotorula, Saccaromyces and Debaryomyces species.

\section{Liquid Assimilation of Carbon and Nitrogen Compounds}

Rij (1984) preferred assimilation tests than the fermentation tests for testing enzyme systems. All tested isolates were able to assimilate D-Sucrose, Raffinose, D-Mannitol, Glycerol, and L-Rhamnose. Majority of the pool (80\%) was able to assimilate D-maltose indicating the presence of the enzyme maltase. The whole pool of isolates was able to assimilate glycerol indicating the presence of glycerol kinase gene (Obasi et al., 2014). All tested isolates were also able to assimilate L-cysteine and L-lysine. Gadaga et al. (2000) reported that $S$. cerevisiae could ferment sucrose, raffinose, glucose and galactose but, unable to utilize lysine. Assimilation of L-lysine indicated the absence of $S$. cerevisiae isolates. This was further confirmed by the genotypic identifications. According to Rij (1984), the inability to utilize nitrate nitrogen is considered to be a valuable tool for characterization of yeast. Some species of Saccharomyces, Kluyveromyces, Pichia and Debaryomycesare unable to utilize nitrates, while the all species of other genera (e.g Hansenula) utilize nitrate. There are some genera in which both nitrate positive and negative species occur (e.g. Candida and 
Trichosporon). Isolates, SLDY_005, SLDY_006, SLDY_015, SLDY_016, SLDY_039, SLDY_044 were unable to assimilate potassium nitrate, therefore could consider as belonging to Saccharomyces, Kluyveromyces, Pichia or Debaryomyces species. However, when these results were compared with the genotypic identifications this was true to all other genera except Pichia isolates which exhibited assimilation of potassium nitrate.

\section{Probiotic potential of the selected isolates}

Species and strain specificity are very important factors (Fijan et al., 2014) when deciding probiotic properties and safety for consumption. Hence, known probiotic species should be considered first for selection. Kluyveromyces species isolated from dairy sources have shown considerable potential in commercial probiotic applications. Further, Pichia and Candida strains are also being studied to a certain extent. Results obtained for the selected isolates of the present study are presented in Table 3. Isolates that grown under all simulated GI conditions were selected as potential probiotics for genotypic identifications. One fourth $(25 \%)$ of yeasts from the initial pool of isolates (80) possessed satisfactory level of probiotic affinities.

(a)

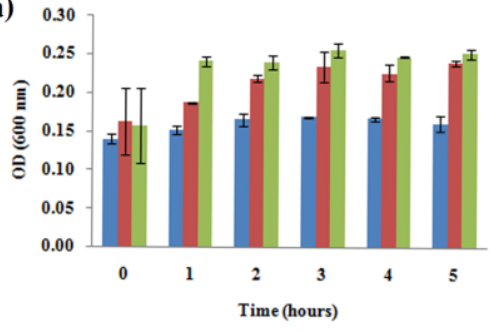

(c)

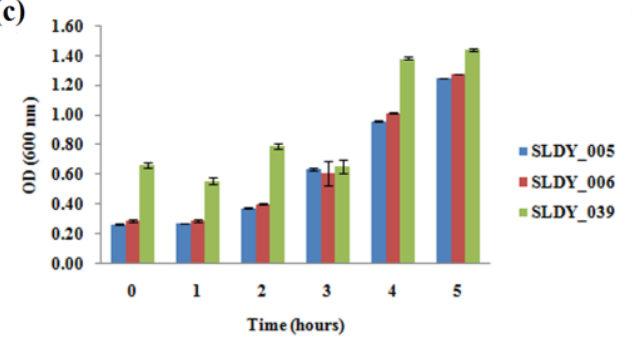

(b)

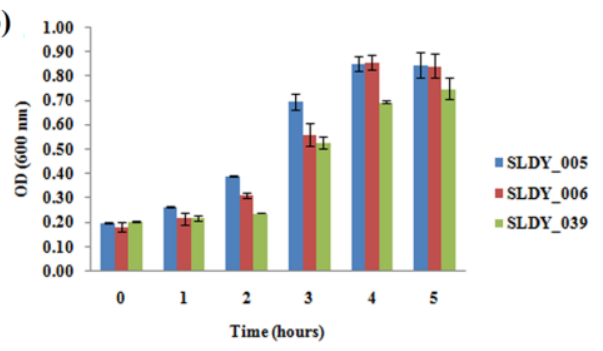

(d)

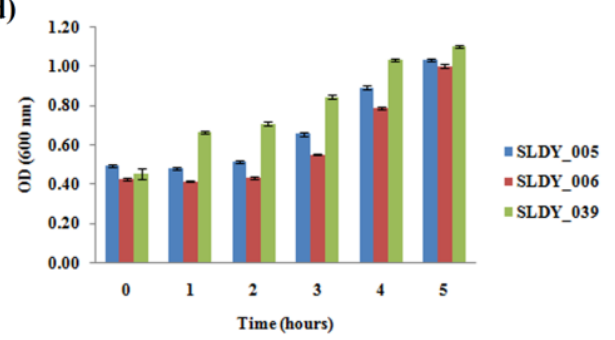

Figure 1. Survival of $K$. marxianus isolates in the presence of acid (a) $\mathrm{pH} 1.5$, (b) $\mathrm{pH} 9.0$ (c) bile $0.3 \%$ and (d) $3.0 \%$ 


\section{Acid and alkaline tolerance}

Survival under a wide range of $\mathrm{pH}$ conditions is an important characteristic of a probiotic yeast to survive in human digestive tract. The selected yeasts were found to grow and survive up to 5 hours under $\mathrm{pH}$ range of 1.5 to 9.0. This confirmed that the isolates can survive in the extreme acidity and alkalinity existing in the stomach and intestines. In agreement with Hamed and Elattar (2013) the viability of many types of yeast decreased at pH 1.5 and growth increased at $\mathrm{pH} 9$ and sustained over 5 hours. They exhibited a very low but, consistent growth compared to initial cell count [unable to grow above one log unit $\left(\log _{10}\right.$ $\mathrm{CFU} / \mathrm{ml}$ )] within 5 hour period. However, at $\mathrm{pH} 9.0$ isolates grew well and initial cell count of $10^{6}$ reached to $10^{7}$ after 5 hours of incubation $\left(\log _{10} \mathrm{CFU} / \mathrm{ml}\right)$. Results obtained for the $K$. marxianus isolates (SLDL_005, SLDL_006 and SLDL_039) identified from the pool is shown in Figure. $1 \mathrm{a}$ and $\mathrm{b}$.

\section{Effects of bile salt on viability}

According to FAO/WHO, it is mandatory to assess bile tolerance for in-vitro selection of probiotic strains (Vinderola et al., 2008). Isolates survived and exhibited gradual increase in cell densities during 5 hour incubation period at $0.3 \%$ and $3 \%$ of bile. As shown in Figure 1. c and d, growth of the yeast (SLDL_005, SLDL_006 and SLDL_039) was not heavily affected by the addition of bile salts as compared to reduced $\mathrm{pH}$. Although, the isolates grew gradually in both bile concentrations, growth was higher at $0.3 \%$. Other researchers have also reported similar results (Sourabh et al,. 2011) in previous studies.

\section{Effects of gastric and pancreatic juice on viability}

Another critical factor that affects the viability of microorganisms during digestion is gastric and pancreatic juices. The obtained results for the above discussed strains are presented in Figure 2. a, b, c and d. At $\mathrm{pH} 8$ isolates showed gradual growth and survival in gastric and pancreatic juices. At $\mathrm{pH} 2$ isolates exhibited a slow growth compared to $\mathrm{pH} 8$ and reached stationary phase earlier (within 3-4 hours).These findings correlate well with the earlier findings of Chelliah et al. (2016) and Díaz-Vergara et al. (2017).

\section{Genotypic identification of most promising probiotic yeast isolates}

Above results revealed that the most promising probiotic yeasts of dairy origin were of Pichia, Candida and Kluyveromyces genera. Considering a threshold of $>95 \%$ similarity to the type strain, 8 different yeast species were identified and listed in table 4. P. kudriavzevii, $K$. marxianus and $C$. tropicalis were the highest 3 probiotic species in frequency of occurrence as shown in Figure 3 . These results well tally with the findings of many researchers previously investigated about the yeast taxonomy in raw milk and dairy products (Fleet, 1990; Wouters, et al., 2002). All identified species of our pool of isolates are there in the Bourdichon's list of beneficial yeasts. Based on species specificity, isolates SLDY_005, SLDY_006 and SLDY_039 which were identified as $K$. marxianus (15\% from the pool) could be considered as the best candidates for further investigations. Maccaferri et al. (2012) reported about the probiotic K. marxianus B0399 (food grade) which has favorably modulated immune response in caco-2 cells, peripheral blood mononuclear cells and exhibited favorable effects on health-promoting bacteria of the genus Bifidobacterium (Bif164). Romanin et al. (2016) reports about anti-inflammatory and anti-oxidative properties of probiotic $K$. marxianus CIDCA 8154. 
Table 2. Biochemical Characteristics of yeast isolates

\begin{tabular}{|c|c|c|c|c|c|c|c|c|c|c|c|c|c|c|}
\hline \multirow[t]{2}{*}{ Isolate } & \multicolumn{3}{|c|}{$\begin{array}{c}\text { Fermentation of } \\
\text { sugars }\end{array}$} & \multicolumn{8}{|c|}{ Carbon assimilation } & \multicolumn{3}{|c|}{ Nitrogen assimilation test } \\
\hline & 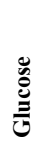 & 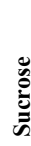 & 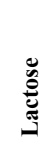 & 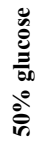 & 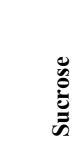 & & 芯 & 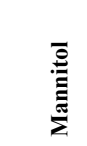 & 苞 & 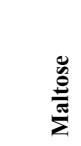 & 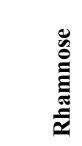 & 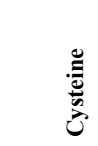 & 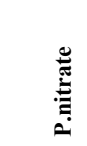 & 节 \\
\hline 1. SLDY_001 & + & + & + & + & + & + & & + & + & + & + & + & + & + \\
\hline 2. SLDY_002 & + & + & + & + & + & + & & + & + & + & + & + & + & + \\
\hline 3. SLDY_003 & + & + & - & + & + & + & & + & + & + & + & + & + & + \\
\hline 4. SLDY_004 & + & + & + & + & + & + & & + & + & + & + & + & + & + \\
\hline 5. SLDY_005 & + & + & + & + & + & + & & + & + & + & + & + & - & + \\
\hline 6. SLDY_006 & + & + & + & + & + & + & & + & + & + & + & + & - & + \\
\hline 7. SLDY_007 & + & + & + & + & + & + & & + & + & + & + & + & + & + \\
\hline 8. SLDY_008 & + & + & + & + & + & + & & + & + & + & + & + & + & + \\
\hline 9. SLDY_011 & + & + & + & + & + & + & & + & + & + & + & + & + & + \\
\hline 10. SLDY__012 & + & - & - & + & + & + & & + & + & + & + & + & + & + \\
\hline 11 .SLDY_013 & + & + & - & + & + & + & & + & + & + & + & + & + & + \\
\hline 12. SLDY_014 & + & + & + & + & + & + & & + & + & + & + & + & + & + \\
\hline 13. SLDY_015 & + & + & - & + & + & + & & + & + & + & + & + & - & + \\
\hline 14. SLDY_016 & + & + & + & + & + & + & & + & + & - & + & + & - & + \\
\hline 15. SLDY_017 & + & - & + & + & + & + & & + & + & + & + & + & + & + \\
\hline 16. SLDY_018 & + & + & + & + & + & + & & + & + & + & + & + & + & + \\
\hline 17. SLDY_019 & + & + & - & + & + & + & & + & + & + & + & + & + & + \\
\hline 18. SLDY_020 & + & + & + & + & + & + & & + & + & + & + & + & + & + \\
\hline 19. SLDY_021 & + & + & + & + & + & + & & + & + & + & + & + & + & + \\
\hline 20. SLDY_022 & + & + & + & + & + & + & & + & + & + & + & + & + & + \\
\hline 21. SLDY_023 & + & - & - & + & + & + & & + & + & + & + & + & + & + \\
\hline 22. SLDY_025 & + & - & - & + & + & + & & + & + & + & + & + & + & + \\
\hline 23. SLDY_026 & + & - & - & - & + & + & & + & + & + & + & + & + & + \\
\hline 24. SLDY_027 & + & - & - & - & + & + & & + & + & + & + & + & + & + \\
\hline 25. SLDY_028 & + & - & - & + & + & + & & + & + & - & + & + & + & + \\
\hline 26. SLDY_029 & + & - & - & - & + & + & & + & + & + & + & + & + & + \\
\hline 27. SLDY_030 & + & + & + & + & + & + & & + & + & - & + & + & + & + \\
\hline 28. SLDY_032 & + & + & + & + & + & + & & + & + & + & + & + & + & + \\
\hline 29. SLDY_035 & + & + & + & + & + & + & & + & + & + & + & + & + & + \\
\hline 30. SLDY_036 & + & + & + & + & + & + & & + & + & + & + & + & + & + \\
\hline 31. SLDY_039 & + & + & + & + & + & + & & + & + & + & + & + & - & + \\
\hline 32. SLDY_040 & + & + & + & + & + & + & & + & + & + & + & + & + & + \\
\hline 33. SLDY_042 & + & + & + & + & + & + & & + & + & + & + & + & + & + \\
\hline 34. SLDY_043 & + & + & + & + & + & + & & + & + & + & + & + & + & + \\
\hline 35. SLDY_044 & + & + & + & + & + & + & & + & + & + & + & + & - & + \\
\hline 36. SLDY_045 & + & + & + & + & + & + & & + & + & - & + & + & + & + \\
\hline
\end{tabular}


Table 3. Survival of selected yeast isolates in the presence of simulated GIT conditions

\begin{tabular}{|c|c|c|c|c|c|c|c|c|c|c|c|c|c|}
\hline \multirow[t]{2}{*}{ Isolate } & \multicolumn{3}{|c|}{ Temperature } & \multicolumn{3}{|c|}{ pH } & \multicolumn{3}{|c|}{ Bile } & \multicolumn{4}{|c|}{ Gastric enzymes } \\
\hline & $\begin{array}{l}0 \\
0 \\
0\end{array}$ & $\begin{array}{l}0 \\
0 \\
\hat{n}\end{array}$ & $\begin{array}{l}0 \\
\text { in } \\
\text { ? }\end{array}$ & $\stackrel{n}{-}$ & $m$ & a & $\begin{array}{l}\stackrel{0}{0} \\
\text { ஸn } \\
0\end{array}$ & $\begin{array}{l}\stackrel{0}{0} \\
\stackrel{0}{0} \\
0\end{array}$ & $\begin{array}{l}\stackrel{0}{8} \\
\text { r. }\end{array}$ & 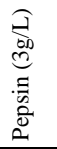 & & 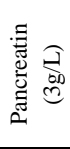 & \\
\hline & & & & & & & & & & $\mathrm{pH} 2$ & $\mathrm{pH} 8$ & $\mathrm{pH} 2$ & $\mathrm{pH} 8$ \\
\hline 1. SLDY 001 & $\sqrt{ }$ & $\sqrt{ }$ & $\sqrt{ }$ & $\sqrt{ }$ & $\sqrt{ }$ & $\sqrt{ }$ & $\sqrt{ }$ & $\sqrt{ }$ & $\sqrt{ }$ & $\sqrt{ }$ & $\sqrt{ }$ & $\sqrt{ }$ & $\sqrt{ }$ \\
\hline 1. & $\sqrt{ }$ & $\sqrt{ }$ & $\sqrt{ }$ & $\sqrt{ }$ & $\sqrt{ }$ & $\sqrt{ }$ & $\sqrt{ }$ & $\sqrt{ }$ & $\sqrt{ }$ & $\sqrt{ }$ & $\sqrt{ }$ & $\sqrt{ }$ & $\sqrt{ }$ \\
\hline 3. SLDY 003 & $\sqrt{ }$ & $\sqrt{ }$ & $\sqrt{ }$ & $\sqrt{ }$ & $\sqrt{ }$ & $\sqrt{ }$ & $x$ & $x$ & $x$ & ND & ND & ND & ND \\
\hline 4. SLDY 004 & $\sqrt{ }$ & $\sqrt{ }$ & $\sqrt{ }$ & $\sqrt{ }$ & $\sqrt{ }$ & $\sqrt{ }$ & $\sqrt{ }$ & $\sqrt{ }$ & $\sqrt{ }$ & $\sqrt{ }$ & $\sqrt{ }$ & $\sqrt{ }$ & $\sqrt{ }$ \\
\hline 5 SLDY 005 & $\sqrt{ }$ & $\sqrt{ }$ & $\sqrt{ }$ & $\sqrt{ }$ & $\sqrt{ }$ & $\sqrt{ }$ & $\sqrt{ }$ & $\sqrt{ }$ & $\sqrt{ }$ & $\sqrt{ }$ & $\sqrt{ }$ & $\sqrt{ }$ & $\sqrt{ }$ \\
\hline 6. SLDY 006 & $\sqrt{ }$ & $\sqrt{ }$ & $\sqrt{ }$ & $\sqrt{ }$ & $\sqrt{ }$ & $\sqrt{ }$ & $\sqrt{ }$ & $\sqrt{ }$ & $\sqrt{ }$ & $\sqrt{ }$ & $\sqrt{ }$ & $\sqrt{ }$ & $\sqrt{ }$ \\
\hline 7. SLDY 007 & $\sqrt{ }$ & $\sqrt{ }$ & $\sqrt{ }$ & $\sqrt{ }$ & $\sqrt{ }$ & $\sqrt{ }$ & $\sqrt{ }$ & $\sqrt{ }$ & $\sqrt{ }$ & $\sqrt{ }$ & $\sqrt{ }$ & $\sqrt{ }$ & $\sqrt{ }$ \\
\hline 8. SLDY_008 & $\sqrt{ }$ & $\sqrt{ }$ & $\sqrt{ }$ & $\sqrt{ }$ & $\sqrt{ }$ & $\sqrt{ }$ & $\sqrt{ }$ & $\sqrt{ }$ & $\sqrt{ }$ & $\sqrt{ }$ & $\sqrt{ }$ & $\sqrt{ }$ & $\sqrt{ }$ \\
\hline 9. SLDY_011 & $\sqrt{ }$ & $\sqrt{ }$ & $\sqrt{ }$ & $x$ & $\sqrt{ }$ & $\sqrt{ }$ & ND & ND & ND & ND & ND & ND & ND \\
\hline 10. SLDY_012 & $\sqrt{ }$ & $\sqrt{ }$ & $\sqrt{ }$ & $x$ & $\sqrt{ }$ & $\sqrt{ }$ & ND & ND & ND & ND & ND & ND & ND \\
\hline 11 .SLDY_013 & $\sqrt{ }$ & $\sqrt{ }$ & $\sqrt{ }$ & $x$ & $\sqrt{ }$ & $\sqrt{ }$ & ND & ND & ND & ND & ND & ND & ND \\
\hline 12. SLDY 014 & $\sqrt{ }$ & $\sqrt{ }$ & $\sqrt{ }$ & $\sqrt{ }$ & $\sqrt{ }$ & $\sqrt{ }$ & $\sqrt{ }$ & $\sqrt{ }$ & $\sqrt{ }$ & $\sqrt{ }$ & $\sqrt{ }$ & $\sqrt{ }$ & $\sqrt{ }$ \\
\hline 13. SLDY & $\sqrt{ }$ & $\sqrt{ }$ & $\sqrt{ }$ & $x$ & $\sqrt{ }$ & $\sqrt{ }$ & ND & ND & ND & ND & ND & ND & ND \\
\hline 14. SLDY_016 & $\sqrt{ }$ & $\sqrt{ }$ & $\sqrt{ }$ & $\sqrt{ }$ & $\sqrt{ }$ & $\sqrt{ }$ & $\sqrt{ }$ & $\sqrt{ }$ & $\sqrt{ }$ & $\sqrt{ }$ & $\sqrt{ }$ & $\sqrt{ }$ & $\sqrt{ }$ \\
\hline 15. SLDY 017 & $\sqrt{ }$ & $\sqrt{ }$ & $\sqrt{ }$ & $\sqrt{ }$ & $\sqrt{ }$ & $\sqrt{ }$ & $\sqrt{ }$ & $\sqrt{ }$ & $\sqrt{ }$ & $\sqrt{ }$ & $\sqrt{ }$ & $\sqrt{ }$ & $\sqrt{ }$ \\
\hline 16. SLDY_018 & $\sqrt{ }$ & $\sqrt{ }$ & $\sqrt{ }$ & $\sqrt{ }$ & $\sqrt{ }$ & $\sqrt{ }$ & $\sqrt{ }$ & $\sqrt{ }$ & $\sqrt{ }$ & $\sqrt{ }$ & $\sqrt{ }$ & $\sqrt{ }$ & $\sqrt{ }$ \\
\hline 17. SLDY_019 & $\times$ & $\sqrt{ }$ & $\sqrt{ }$ & $\sqrt{ }$ & $x$ & $x$ & ND & ND & ND & ND & ND & ND & ND \\
\hline 18. SLDY_020 & $\sqrt{ }$ & $\sqrt{ }$ & $\sqrt{ }$ & $\sqrt{ }$ & $\sqrt{ }$ & $\sqrt{ }$ & $\sqrt{ }$ & $\sqrt{ }$ & $x$ & ND & ND & ND & ND \\
\hline 19. SLDY 021 & $\sqrt{ }$ & $x$ & $x$ & $\sqrt{ }$ & $\sqrt{ }$ & $\sqrt{ }$ & $x$ & $x$ & $x$ & ND & ND & ND & ND \\
\hline 20. SLDY 022 & $\sqrt{ }$ & $\sqrt{ }$ & $\sqrt{ }$ & $\sqrt{ }$ & $\sqrt{ }$ & $\sqrt{ }$ & $\sqrt{ }$ & $\sqrt{ }$ & $\sqrt{ }$ & $\sqrt{ }$ & $\sqrt{ }$ & $\sqrt{ }$ & $\sqrt{ }$ \\
\hline 21. SLDY_023 & $\times$ & $\sqrt{ }$ & $\sqrt{ }$ & $x$ & $x$ & $\sqrt{ }$ & ND & ND & ND & ND & ND & ND & ND \\
\hline 22. SLDY_025 & $x$ & $\sqrt{ }$ & $\times$ & $x$ & $\sqrt{ }$ & $\sqrt{ }$ & ND & ND & ND & ND & ND & ND & ND \\
\hline 23. SLDY_026 & $\times$ & $\sqrt{ }$ & $\sqrt{ }$ & $x$ & $\sqrt{ }$ & $\sqrt{ }$ & ND & ND & ND & ND & ND & ND & ND \\
\hline 24. SLDY_027 & $\sqrt{ }$ & $\sqrt{ }$ & $x$ & $\sqrt{ }$ & $\sqrt{ }$ & $\sqrt{ }$ & $x$ & $x$ & $x$ & ND & ND & ND & ND \\
\hline 25. SLDY_028 & $x$ & $\sqrt{ }$ & $x$ & $x$ & $\sqrt{ }$ & $\sqrt{ }$ & ND & ND & ND & ND & ND & ND & ND \\
\hline 26. SLDY_029 & $\sqrt{ }$ & $\sqrt{ }$ & $\sqrt{ }$ & $x$ & V & $\sqrt{ }$ & ND & ND & ND & ND & ND & ND & ND \\
\hline 27. SLDY_030 & $\sqrt{ }$ & $\sqrt{ }$ & $\sqrt{ }$ & $\sqrt{ }$ & $\sqrt{ }$ & $\sqrt{ }$ & $\sqrt{ }$ & $\sqrt{ }$ & $\sqrt{ }$ & $\sqrt{ }$ & $\sqrt{ }$ & $\sqrt{ }$ & $\sqrt{ }$ \\
\hline 28. SLDY_032 & $\sqrt{ }$ & $x$ & $\times$ & $x$ & $\sqrt{ }$ & $\sqrt{ }$ & ND & ND & ND & ND & ND & ND & ND \\
\hline 29. SLDY_035 & $\sqrt{ }$ & $\sqrt{ }$ & $\sqrt{ }$ & $\sqrt{ }$ & $\sqrt{ }$ & $\sqrt{ }$ & $\sqrt{ }$ & $\sqrt{ }$ & $\sqrt{ }$ & $\sqrt{ }$ & $\sqrt{ }$ & $\sqrt{ }$ & $\sqrt{ }$ \\
\hline & $\sqrt{ }$ & $\sqrt{ }$ & $\sqrt{ }$ & $\sqrt{ }$ & $\sqrt{ }$ & $\sqrt{ }$ & $\sqrt{ }$ & $\sqrt{ }$ & $\sqrt{ }$ & $\sqrt{ }$ & $\sqrt{ }$ & $\sqrt{ }$ & $\sqrt{ }$ \\
\hline 31. SLDY_039 & $\sqrt{ }$ & $\sqrt{ }$ & $\sqrt{ }$ & $\sqrt{ }$ & $\sqrt{ }$ & $\sqrt{ }$ & $\sqrt{ }$ & $\sqrt{ }$ & $\sqrt{ }$ & $\sqrt{ }$ & $\sqrt{ }$ & $\sqrt{ }$ & $\sqrt{ }$ \\
\hline 32. SLDY_040 & $\times$ & $\sqrt{ }$ & $\sqrt{ }$ & $\sqrt{ }$ & $\sqrt{ }$ & $\sqrt{ }$ & $\sqrt{ }$ & $\sqrt{ }$ & $x$ & ND & ND & ND & ND \\
\hline 33. SLDY_042 & $\sqrt{ }$ & $\sqrt{ }$ & $\sqrt{ }$ & $\sqrt{ }$ & $\sqrt{ }$ & $\sqrt{ }$ & $\sqrt{ }$ & $\sqrt{ }$ & $\sqrt{ }$ & $\sqrt{ }$ & $\sqrt{ }$ & $\sqrt{ }$ & $\sqrt{ }$ \\
\hline 34. SLDY_043 & $\sqrt{ }$ & $\sqrt{ }$ & $\sqrt{ }$ & $\sqrt{ }$ & $\sqrt{ }$ & $\sqrt{ }$ & $\sqrt{ }$ & $\sqrt{ }$ & $\sqrt{ }$ & $\sqrt{ }$ & $\sqrt{ }$ & $\sqrt{ }$ & $\sqrt{ }$ \\
\hline 35. SLDY_044 & $\sqrt{ }$ & $\sqrt{ }$ & $\sqrt{ }$ & $\sqrt{ }$ & $\sqrt{ }$ & $\sqrt{ }$ & $\sqrt{ }$ & $\sqrt{ }$ & $\sqrt{ }$ & $\sqrt{ }$ & $\sqrt{ }$ & $\sqrt{ }$ & $\sqrt{ }$ \\
\hline 36. SLDY_045 & $\sqrt{ }$ & $\sqrt{ }$ & $\sqrt{ }$ & $\sqrt{ }$ & $\sqrt{ }$ & $\sqrt{ }$ & $\sqrt{ }$ & $\sqrt{ }$ & $\sqrt{ }$ & $\sqrt{ }$ & $\sqrt{ }$ & $\sqrt{ }$ & $\sqrt{ }$ \\
\hline
\end{tabular}

ND: Not Done 

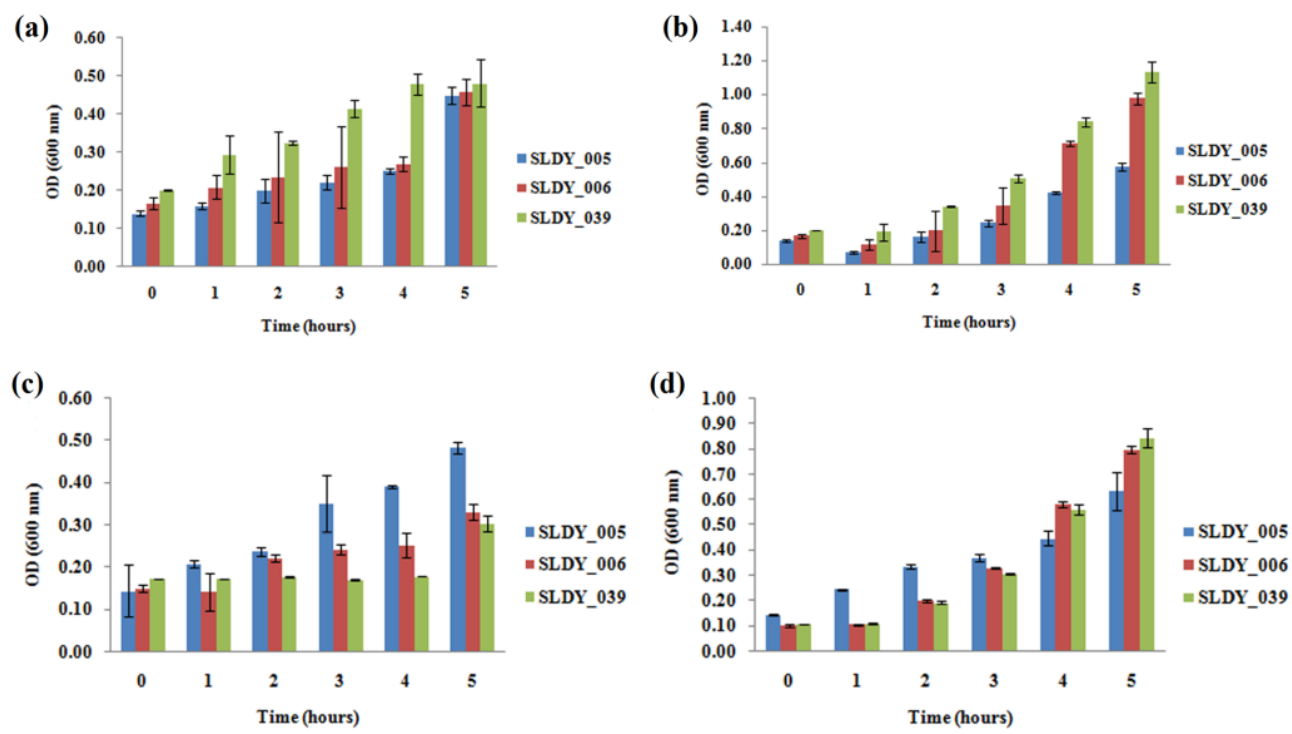

Figure 2. Survival of $K$. marxianus isolates in the presence of pancreatin (a) pH 2, (b) pH 8.0, (c) pepsin pH 2 and (d) pH 8.0

Pichia is the predominant probiotic genera identified from the pool (55\%) which also had exhibited probiotic potential and safety in previous studies. Greppi et al. (2017) has tested and confirmed the probiotic potential of P.kudriavzevii strains and their ability to enhance folate content of traditional cereal-based African fermented food. Chelliah et al. (2016) has evaluated and confirmed the antimicrobial activity and probiotic properties of P.kudriavzevii isolated from frozen idli batter and Ogunremi et al. (2015) has developed a cereal-based functional food using cereal-mix substrate fermented with probiotic strain - P.kudriavzevii OG32.Therefore, Pichia isolates could consider as the second priority for further investigations.

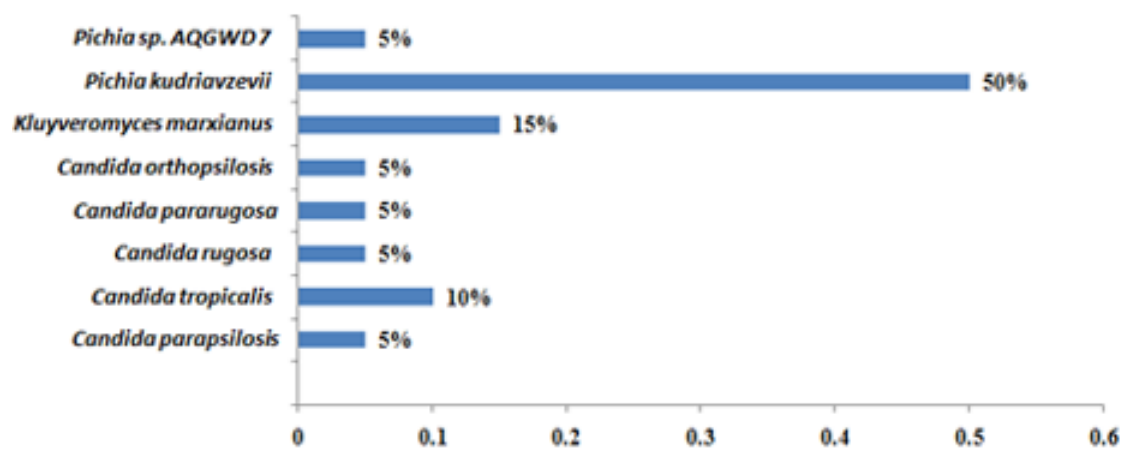

Figure 3. Abundance of potentially probiotic yeast species isolated from dairy sources of Sri Lanka 
Table 4. Potentially probiotic yeast species isolated from dairy sources of Sri Lanka with \% similarity to type strain

\begin{tabular}{llll}
\hline Code & & Identity & Similarity to type strain \\
\hline SLDY_001 & Curd & Pichia kudriavzevii isolate C12 & $100 \%$ \\
SLDY_002 & Curd & Pichia kudriavzevii strain IFM 64555 & $100 \%$ \\
SLDY_004 & Curd & Pichia kudriavzevii strain IFM 64555 & $100 \%$ \\
SLDY_005 & Curd & Kluyveromyces marxianus CBS 5673 & $100 \%$ \\
SLDY_006 & Curd & Kluyveromyces marxianus CBS 5673 & $100 \%$ \\
SLDY_007 & Curd & Pichia kudriavzevii IFM 56882 & $100 \%$ \\
SLDY_008 & Curd & Pichia sp. AQGWD 7 & $99 \%$ \\
SLDY_014 & Curd & Candida tropicalis SBKS 3 & $100 \%$ \\
SLDY_016 & Curd & Candida parapsilosis isolate S22811 & $100 \%$ \\
SLDY_017 & Curd & Pichia kudriavzevii strain IFM 64555 & $100 \%$ \\
SLDY_018 & Curd & Pichia kudriavzevii strain IFM 64555 & $100 \%$ \\
SLDY_022 & Curd & Pichia kudriavzevii isolate H-237 & $96 \%$ \\
SLDY_030 & Curd & Pichia kudriavzevii strain B187B & $100 \%$ \\
SLDY_035 & Raw cows' milk & Pichia kudriavzevii strain YB-25 & $100 \%$ \\
SLDY_036 & Raw cows' milk & Pichia kudriavzevii strain B187B & $100 \%$ \\
SLDY_039 & Raw cows' milk & Kluyveromyces marxianus strain CBS 1555 & $100 \%$ \\
SLDY_042 & Raw cows' milk & Candida rugosa strain CBS 613 & $100 \%$ \\
SLDY_043 & Raw cows' milk & Candida tropicalis LEM123 & $99 \%$ \\
SLDY_044 & Raw cows' milk & Candida pararugosa strain M172B & $100 \%$ \\
SLDY_045 & Raw cows' milk & Candida orthopsilosis strain IFM55182 & $100 \%$ \\
\hline & & &
\end{tabular}

\section{CONCLUSIONS}

This investigation provides a theoretical basis for probiotic yeast diversity of Sri Lankan dairies. This might be an attractive solution to the steadily increasing demands of food manufacturers looking for probiotics with viability under extreme conditions. Identified isolates could be useful for probiotic strain selection, manufacturing dairy products for lactose intolerant people, production of fermented foods with high concentrations of sugar, single cell proteins (SCP) and bio ethanol production from whey, and production of functional ingredients for food and pharmaceutical industries. P.kudriavzevii and $K$. marxianus (65\% from the total) were the best probiotics identified therefore, worth to study further to establish as commercial probiotics. Moreover, remaining species of the pool $(P$. AQGWD 7, C.pararugosa, C. tropicalis, C. metapsilosis, C. rugosa, C. orthopsilosis) also could consider as promising candidates for local bio processing industries. 


\section{ACKNOLEDGEMENT}

The authors wish to acknowledge the financial support provided by the Industrial Technology Institute, Colombo, Sri Lanka (TG 15/117) and National Science Foundation Grant (RG/2016/AG/02).

\section{REFERENCES}

Aswathy, R.G., Ismail, B., John, R.P. and Nampoothiri, K.M. (2008). Evaluation of the probiotic characteristics of newly isolated lactic acid bacteria. Applied Biochemistry and Biotechnol.151, 244-255.

Barnett, Y.A., Payne, R.W. and Yarrow, D. (2000). Yeasts: characteristics and identification, Cambridge Uni. Press, U.K, pp. 25-35.

Bourdichon, F., Casaregola, S., Farrokh, C., Frisvad, J.C., Gerds, M.L., Prajapati, J.P., Seto, Y., Schure, E.T., Boven, A.V., Vankerckhoven, V., Zgoda, A., Tuijtelaars S. and Hansen, E.B. (2012). Food fermentations: Microorganisms with technological beneficial use. Int. J. of Food Microbiol. 154, 87-97.

Chelliah, R.P., Ramakrishnan, S.R., Prabhu, P.R. and Antony, U. (2016). Evaluation of antimicrobial activity and probiotic properties of wild-strain Pichia kudriavzevii isolated from frozen idli batter. Yeast special Issue; 33: 385-401.

Christensen, W. B. (1946). Urea decomposition as a means of differentiating Proteus and paracolon cultures from each other and from Salmonella and Shigella types. J. of Bacteriol. 52, 461-466.

Díaz-Vergara, L., Pereyra, C.M., Montenegro, M., Pena, G.A., Aminahuel, C.A. and Cavaglieri, L.R. (2017). Encapsulated whey-native yeast Kluyveromyces marxianus as a feed additive for animal production. Food Additives and Contaminants. 34, 750-759.

Ebabhi, A.M., Adekunle, A.A., Okunowo, W.O. and Osuntoki, A.A. (2013). Isolation and characterization of yeast strains from local food crops. J. of Yeast and Fungal Research. 4(4), $38-43$.

FAO/WHO (2002). Guidelines for the evaluation of probiotics in food. Food and Agriculture Organization of the United Nations and World Health Organization working group report, London Ontario, Canada.

Fijan, S. (2014). Microorganisms with claimed probiotic properties: An overview of recent literature. Int. J. Environ. Res. Public Health. 11, 4745-4767.

Fleet, G.H. (1990). Yeasts in dairy products- A review. J. Appl. Bacteriol. 68, 199-211.

Gadaga, T.H., Mutukumira, A.N. and Narvhus J.A. (2000). Enumeration and identification of yeasts isolated from Zimbabwean traditional fermented milk. Int. Dairy J. 10, 459-466. 
Gana, N.H.T., Mendoza, B.C. and Monsalud, R.G. (2014). Isolation, screening and characterization of yeasts with amyloytic, lipolytic, and proteolytic activities from the surface of Philippine bananas (musa spp.), Philippine J.of Sci.143 (1), 81-87.

Greppi, A., Saubade, F., Botta, C., Humblot, C., Guyot, J.P. and Cocolin, L. (2017). Potential probiotic Pichia kudriavzevii strains and their ability to enhance folate content of traditional cereal-based African fermented food. Food Microbiol. 62, 169-177.

Hamed, E. and Elattar, A. (2013). Identification and some probiotic potential of lactic acid bacteria isolated from Egyptian camels milk. Life Sci. J. 10(1), 1952-1961.

Jakobsena, M. and Narvhu, J. (1996). Yeasts and their possible beneficial and negative effects on the quality of dairy products. Dairy J.6, 755-768.

Kurtzman, C.P., Fell, J.W. and Boekhout, T. (2011). The Yeasts: A Taxonomic Study, $5^{\text {th }}$ Edn., Elsevier Science, Burlington, U.K.

Li, Y., Liu, T. and He, G. (2015). Isolation and Identification of Yeasts from Tibet Kefir. Ad. J. of Food Sci. and Technol. 7(3): 199-203.

Maccaferri, S., Klinder, A., Brigidi, P., Cavina, P. and Costabile, A. (2012). Potential Probiotic Kluyveromyces marxianus B0399 modulates the immune response in caco-2 cells and peripheral blood mononuclear cells and impacts the human gut microbiota in an in-vitro colonic model system. Appl. Environ. Microbiol, 78 (4) 956-964.

Nahvi, I. and Moeini, H. (2004). Isolation and identification of yeast strains with high $\beta$ galactosidase activity from dairy products. Biotechnol. 3: 35-40.

Nayak S.K. (2011). Probiotics, Microbiology Monographs. 21, 29-55.

Obasi, B.C., Whong, C.M.Z., Ado, S.A. and Abdullah, I.O. (2014), Isolation and Identification of yeast associated with fermented orange juice. Int. J. of Engineering and Sci.3, 9, 64-69.

Oda, Y. and Ouchi, K. (2000). Saccharomyces, Encyclopedia of food microbiology. Richard K., Robinson C.A., Batt P. and Patel D. (Ed.) Academic press, Londan, U.K. 3, 1907-1927.

Ogunremi, O.R., Agrawal, R. and Sanni, A.I. (2015). Development of cereal-based functional food using cereal-mix substrate fermented with probiotic strain - Pichia kudriavzevii OG32. Food Sci. and Nutrition, Wiley Periodicals, Inc. pp.486-489.

Rij, N.J.W.K. (1984). The yeasts. Elsevier Science Publishers B.V., Amsterdam. pp.75-95.

Romanin, D.E., Llopis, S., Genovés, S., Martorell, P., Ramón, V.D., Garrote, G.L. and Rumbo, M. (2016). Probiotic yeast Kluyveromyces marxianus CIDCA 8154 shows antiinflammatory and anti-oxidative stress properties in in-vivo models. Beneficial Microbes. 8393.

Rutherford, J.C. (2014). The emerging role of urease as a general microbial virulence factor. PLOS Pathogens | www.plospathogens.org. 
Sourabh, A., Kanwar, S.S. and Sharma, O.P. (2011). Screening of indigenous yeast isolates obtained from traditional fermented foods of Western Himalayas for probiotic attributes. J. of Yeast and Fungal Research. 2(8), 117 - 126.

Spinnler, H.E. and Corrieu, G. (1989). Automatic method to quantify starter activity based on $\mathrm{pH}$ measurement. J. of Dairy Research. 56, 755-764.

Vinderola, G., Capellini, B., Villarreal, F., Suárez, V., Quiberoni, A. and Reinheimer, J. ( 2008). Usefulness of a set of simple in-vitro tests for the screening and identification of probiotic candidate strains for dairy use. LWT - Food Sci. and Technology, 41, 1678-1688.

Walker, D. K. and Gilliland, S. E. (1993). Relationships among bile tolerance, bile salt deconjugation and assimilation of cholesterol by Lactobacillus acidophilus. J. of Dairy Sci., 7695.

Wouters, J.T.M., Eman H.E., Jeroen, A. and Smit H.G. (2002). Microbes from raw milk for fermented dairy products, Int. Dairy J, 12, 91-109. 